\title{
Le problène
}

\section{des pompes-turbines}

\section{vu parlle maîre d'oevire}

\section{Points de vue lechnique el économique}

\section{Jeanmoël Plichon}

Contrôleur Général à E.D.F., Direction de l'Equipement

\section{André Lescure}

Chargé de Mission à E.D.F., Direction de l'Equipement

\section{Antoine Dumaine}

Attaché à E.D.F., Direction de l'Equipement

\section{Daniel Simonnol}

Ingénieur à E.D.F., Direction de l'Equipement

\section{Avantages et inconvénients des pompes-turbines par rapport aux groupes ternaires}

Les groupes binaires comportent comme les groupes ternaires une seule machine électrique fonctionnant tantôt en alternateur, tantôt en moteur, mais ils se différencient de ces groupes par la partie hydraulique : alors que les groupes ternaires sont équipés d'une turbine et d'une pompe, les groupes binaires ne possèdent qu'une seule machine hydraulique appelée pompeturbine et conçue pour fonctionner soit en turbine soit en pompe par inversion de son sens de rotation.

Les avantages et les inconvénients de ces groupes désignés couramment sous le nom de "groupes réversibles" par rapport aux groupes ternaires résultent de cette différence.

\section{Avantages}

a) La suppression d'une machine hydraulique, de ses conduites, de ses vannes et de tout son matériel annexe entraîne une réduction importante du volume de la centrale et, bien que les pompes-turbines aient une bâche plus encombrante que les turbines classiques et qu'elles nécessitent un dispositif de lancement en pompe, le bilan est toujours en leur faveur ; le gain réalisé est particulièrement important pour les hautes chutes qui imposent dans la solution ternaire l'emploi de turbines Pelton : l'implantation de ces turbines au-dessus du niveau aval le plus élevé et celle des pompes qui exigent une pression d'aspiration importante conduisent en effet à des groupes ayant une très grande longueur d'arbre et par suite à des centrales très volumineuses. Comparaison faite pour l'usine de $\mathrm{La}$ Coche:

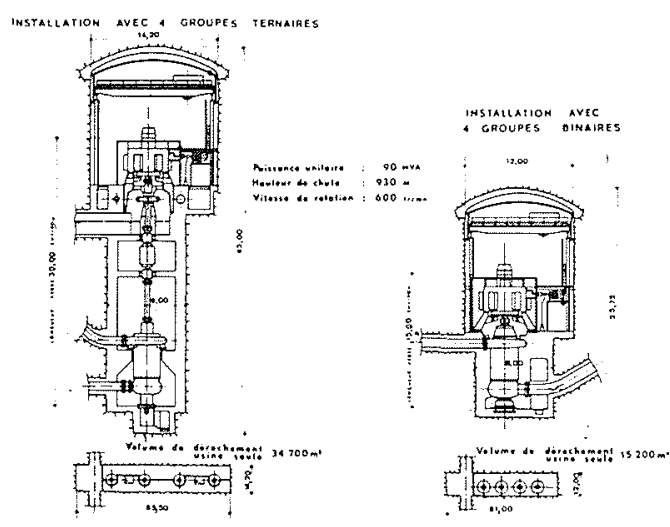

b) La suppression du matériel indiqué ci-dessus et celle du système de débrayage de la pompe des groupes ternaires font faire par ailleurs sur la fourniture une économie nettement supérieure au prix parfois élevé du matériel nécessaire au démarrage en pompe des groupes binaires.

Les comparaisons de prix établies respectivement en 1969 et 1976 pour l'usine de La Coche et celle de 
Moralets située dans les Pyrénées espagnoles sont indiquées ci-après :

\begin{tabular}{|c|c|c|}
\hline \multicolumn{3}{|c|}{$\begin{array}{c}\text { DEVIS LA COCHE } \\
\text { Solution Groupes Ternaires } \\
\text { Devis Bases juillet } 1964\end{array}$} \\
\hline Désignation & $\begin{array}{c}\text { Prix en } 10^{6} \mathrm{~F} \\
\text { T.T.C. }\end{array}$ & Niveau \\
\hline 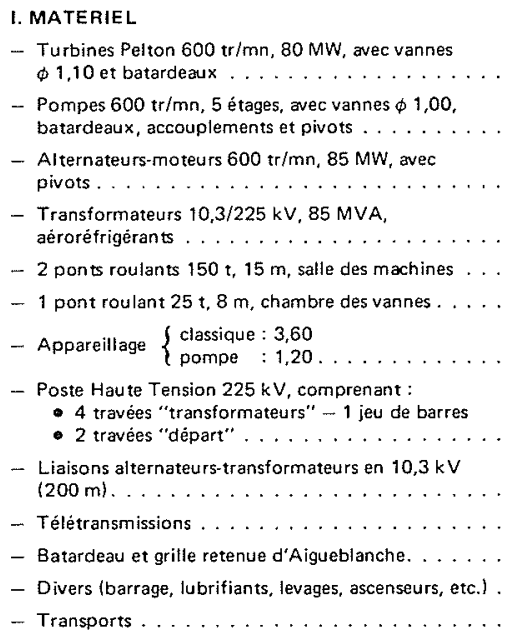 & $\begin{array}{l}10,10 \\
18,00 \\
14,80 \\
6,90 \\
1,20 \\
0,15 \\
4,80 \\
\\
2,80 \\
0,50 \\
0,70 \\
0,40 \\
1,35 \\
1,10\end{array}$ & \\
\hline $\begin{array}{l}\text { Total. } \ldots \ldots \\
\text { 11. GENIE CIVIL } \\
\text { Prix approché } \ldots \ldots \ldots \ldots \ldots \ldots \ldots \ldots \ldots\end{array}$ & 62,80 & 100 \\
\hline TOTAL Matériel et Génie Civil $\ldots \ldots \ldots$ & 84,80 & 100 \\
\hline
\end{tabular}

\begin{tabular}{|c|c|c|}
\hline \multicolumn{3}{|c|}{$\begin{array}{c}\text { DEVIS LA COCHE } \\
\text { Solution Groupes Binaires } \\
\text { Devis Bases juillet } 1964\end{array}$} \\
\hline Désignation & $\begin{array}{c}\text { Prix en } 10^{6} \mathrm{~F} \\
\text { T.T.C. }\end{array}$ & Niveau \\
\hline 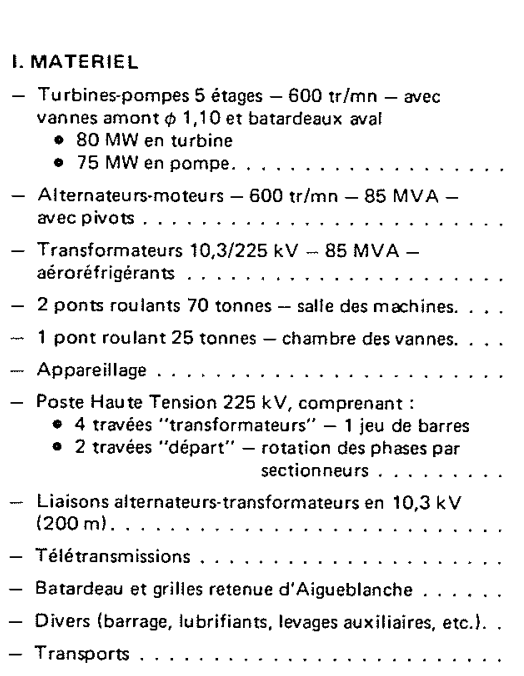 & $\begin{array}{r}16,60 \\
15,00 \\
7,00 \\
0,65 \\
0,15 \\
3,20\end{array}$ & $\begin{array}{l}\text { par } \\
\text { rapport } \\
\text { solution } \\
\text { groupes } \\
\text { ternaires }\end{array}$ \\
\hline $\begin{array}{l}\text { Total. } \ldots \ldots \\
\text { 11. GENIE CIVIL } \\
\text { Prix approché } \ldots \ldots \ldots \ldots \ldots \ldots \ldots \ldots\end{array}$ & 49 & 78 \\
\hline TOTAL Matériel et Génie Civil $\ldots \ldots \ldots \ldots \ldots$ & 65 & 77 \\
\hline
\end{tabular}

\begin{tabular}{|c|c|c|c|c|}
\hline \multicolumn{5}{|c|}{$\begin{array}{c}\text { DEVIS MORALETS } \\
\text { (châte de } 740 \mathrm{~m} \text { dans les Pyrénées Espagnoles } \\
3 \text { groupes ternaires et } 3 \text { groupes binaires } \\
\text { en } 10^{6} \mathrm{~F} \text { Bases } 1.1 .1974\end{array}$} \\
\hline \multirow[b]{3}{*}{ 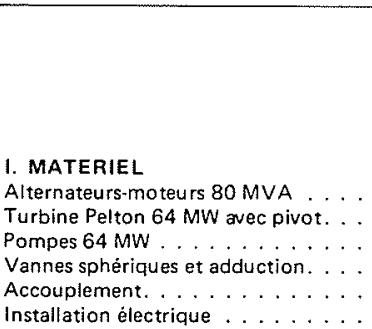 } & \multicolumn{2}{|c|}{3 groupes ternaires } & \multicolumn{2}{|c|}{3 groupes binaires } \\
\hline & $\ln 10^{6} \mathrm{~F}$ & Niveau & en $10^{6} \mathrm{~F}$ & Niveau \\
\hline & $\begin{array}{r}16,2 \\
9,8 \\
20,7 \\
5,2 \\
2,0 \\
3,6\end{array}$ & & $\begin{array}{c}17 \\
0 \\
20,7 \\
4,5 \\
0 \\
3,4\end{array}$ & $\begin{array}{l}\text { par } \\
\text { rapport } \\
\text { solution } \\
\text { groupes } \\
\text { ternaires }\end{array}$ \\
\hline $\begin{array}{l}\text { Total Matériels non communs } \\
\text { aux } 2 \text { solutions } \ldots \ldots \ldots \ldots \ldots \\
\text { II. GENIE cIVIL } \\
\text { Prix approché } \ldots \ldots \ldots \ldots \ldots\end{array}$ & $\begin{array}{l}57,5 \\
14\end{array}$ & 100 & $\begin{array}{l}45,6 \\
10\end{array}$ & 80 \\
\hline TOTAL Matériel et Génie Civil & 71,5 & 100 & 55,6 & 77 \\
\hline
\end{tabular}

Elles font ressortir les économies suivantes en faveur des groupes binaires :

$\begin{array}{lll}\text { Pour La Coche : } & \text { sur le génie civil } & 27 \% \\ & \text { sur le matériel } & 22 \% \\ & \text { sur l'ensemble } & 23 \% \\ \text { Pour Moralets : } & \text { sur le génie civil } & 29 \% \\ & \text { sur le matériel } & 20 \% \\ & \text { sur l'ensemble } & 23 \%\end{array}$

\section{Inconvénients}

a) $\mathrm{La}$ roue des pompes-turbines étant un compromis entre celle d'une pompe et celle d'une turbine, il en résulte un certain nombre d'inconvénients tels que :

- perte de rendement en turbine de quelques pour-cent, l'écoulement ayant lieu dans des conduits de faible convergence ;

- difficulté pour ajuster les caractéristiques dans les deux modes de fonctionnement : le rendement maximal en turbine ne correspond pas à la même hauteur de chute que le rendement maximal en pompe et la zone de recouvrement des hauteurs pour lesquelles les rendements sont élevés en pompe et en turbine est parfois assez étroite.

Cette difficulté à laquelle s'ajoute celle de connaître a priori la position des charges critiques, c'est-à-dire les zones exactes d'utilisation des caractéristiques, conduit en général le maître d'œuvre à laisser une certaine liberté au constructeur dans le choix du rapport débit turbiné/ débit pompé, ce qui entraîne une incertitude importante dans la définition du cycle de fonctionnement des installations au stade de l'étude économique. Voici, à titre indicatif, la valeur du rapport $Q_{t} \max / Q_{p} \max$ mesuré ou prévu dans nos usines :

$\begin{array}{ll}\text { Vouglans } & \frac{69}{68,6} \# 1 \\ \text { Sainte Croix } & \frac{87,6}{70,6}=1,24\end{array}$




$\begin{array}{ll}\text { Revin } & \frac{96}{74}=1,30 \\ \text { Arc-Isère } & \frac{114}{89,1}=1,28 \\ \text { Montézic } & \frac{63}{51,5}=1,22 \\ \text { La Coche } & \frac{9,6}{8,7}=1,10\end{array}$

On voit qu'il y a des différences assez importantes.

En ce qui concerne le débit maximal en pompe, il faut signaler que l'on constate actuellement chez les constructeurs une certaine difficulté à prévoir sa valeur; ceci est dû à la distorsion prototype-modèle et peut avoir des conséquences importantes en matière de définition de la puissance maximale de l'alternateur-moteur, de cavitation et de régimes transitoires. Les dépassements constatés par rapport aux valeurs garanties sont les suivants :

à Revin : $10 \%$ sur le débit et sur la puissance

à Vouglans : $5,4 \%$ sur le débit et $3 \%$ sur la puissance.

b) Surpression plus élevée dans les conduites haute pression. Cette surpression est en effet provoquée par la roue elle-même après un déclenchement en turbine et dépend donc du diamètre d'entrée qui est plus grand que celui d'une turbine Francis de même vitesse spécifique ( $40 \%$ environ), et des angles de l'aubage qui sont plus petits.

c) Les groupes binaires ne peuvent pas démarrer en pompe à l'aide de leur machine hydraulique ; pour les amener à la vitesse de synchronisme, il faut utiliser soit leur machine électrique soit un dispositif spécial de lancement.

d) Les roues des groupes réversibles sont de construction assez difficile à cause de leurs canaux qui sont plus longs que ceux des groupes classiques.

e) Des difficultés particulières de mise au point peuvent se présenter : ainsi un accident grave s'est produit à Revin sur le groupe $n^{\circ} 2$ au cours du premier essai de prise du débit en pompage (après couplage du groupe et renoyage de la roue) ; dès la disparition de la contrainte de fermeture des directrices, 3 doigts de sécurité ont été cisaillés et les directrices correspondantes, devenues libres, ont été happées par l'effet d'écope et entraînées jusqu'au contact de la roue, les fixations du support des butées ayant cédé. Ni les essais sur modèle réduit sous pression réelle, ni une exploitation industrielle de ce modèle comme groupe auxiliaire n'ont permis de prévoir cet incident : on attendait pour les petites ouvertures une forte tendance à la fermeture due à la dépression au col des canaux entre directrices, et qui devait appuyer chaque levier de directrice sur sa butée sans sollicitation du doigt de sécurité ; c'est une violente tendance à l'ouverture qui s'est produite.

Le processus du phénomène vibratoire source de l'incident reste sujet à débat :
- Le constructeur pense qu'on a eu affaire à la vibration normale de la pale sollicitée périodiquement par le passage des canaux de la roue et que cette vibration a été amplifiée par une résonance malencontreuse de la fréquence mal connue de torsion sur le premier harmonique de la fréquence du passage des canaux : des essais de vibration d'une directrice dans l'eau avaient laissé entrevoir une diminution de $10 \%$ de la fréquence propre de torsion par effet de la masse ajoutée de l'eau sollicitée par cette vibration ; des mesures faites après l'incident sur les directrices dans leur cadre d'installation réel ont indiqué un abaissement plus important (25 à $30 \%$ ).

- Autre explication du phénomène : l'écoulement entre directrices aurait engendré lui-même la vibration; la surlongueur des directrices destinée à améliorer la récupération des vitesses de l'eau en pompage ne serait d'ailleurs pas étrangère au phénomène qui met en cause l'élasticité de l'attelage de chaque directrice et les jeux mécaniques aux différentes articulations, c'est-à-dire la possibilité de mouvements relatifs de 2 directrices voisines.

- Les principales modifications faites par le constructeur ont été les suivantes:

- renforcement du mécanisme de commande des directrices ;

- adjonction à chaque directrice d'un vérin rotatif ayant pour fonction :

- de supprimer l'influence des jeux par introduction d'un couple antagoniste ;

- de freiner la rotation en cas de rupture du doigt de sécurité ;

- d'augmenter substantiellement la fréquence de torsion de la directrice ;

- de créer un effet d'amortissement lors des transitoires (en turbinage et en pompage);

- diminution du diamètre de la roue pour éviter la possibilité de contact avec les directrices

- réduction de la longueur des directrices aux deux extrémités pour diminuer les variations de tendance hydraulique lors de l'ouverture en pompe au voisinage de la pleine fermeture et pour réduire les efforts de manœuvre lors de l'ouverture en pompe ;

- perfectionnement du système des doigts de rupture par une mise en contrainte et une élimination du jeu.

f) Les dispositifs de graissage des paliers et des pivots ainsi que le système de ventilation des alternateurs doivent être adaptés au double sens de rotation.

g) Pour les chutes supérieures à $500 \mathrm{~m}$ environ, les pompes-turbines comportent plusieurs étages et peuvent difficilement de ce fait être équipées de directrices orientables. Toutes celles qui sont en service ou en cours de construction (La Coche, Chiotas-Piastra) sont à distributeur fixe ce qui ne permet pas de régler leur puissance d'une façon continue et les rend donc inaptes au réglage de la fréquence du réseau.

Des études sont cependant en cours en vue de réaliser des pompes-turbines à 2 étages équipés chacun de directrices mobiles qui permettraient de repousser à $1000 \mathrm{~m}$ environ la limite d'utilisation des groupes réglables. 


\section{Problèmes particuliers aux pompes-turbines}

\section{a) Choix du type de démarrage en pompe}

Ce choix est fonction de plusieurs critères:

- nombre de groupes à démarrer

- caractéristiques des groupes (inertie, couple moteur, couple résistant, réactances, etc.)

- caractéristiques đu réseau électrique (tension, puissance de court-circuit)

- facilité d'installation du matériel de lancement et éventuellement des stations de dénoyage des roues.

Les différents procédés de démarrage utilisés ou prévus en France actuellement sont les suivants :

- Démarrages par l'alternateur-moteur lui-même :

- par le procédé asynchrone à pleine tension : groupe de Vouglans (57 MW - $150 \mathrm{tr} / \mathrm{mn}$ ) (en secours), roue noyée

groupe IV de Revin (180 MW - $300 \mathrm{tr} / \mathrm{mn}$ ) roue dénoyée (ou éventuellement roue noyée)

groupes de La Coche (80 MW - $600 \mathrm{tr} / \mathrm{mn}$ ) roue noyée

groupe de Sainte-Croix (58 MW - $166 \mathrm{tr} / \mathrm{mn}$ ) (en secours), roue noyée.

- par le procédé asynchrone à tension réduite : groupes d'Arc-Isère (243 MW - $300 \mathrm{tr} / \mathrm{mn})$, roue dénoyée

- Démarrages par un moteur auxiliaire asynchrone accouplé sur le même arbre que le groupe:

- groupes I, II et III de Revin (180 MW - $300 \mathrm{tr} / \mathrm{mn})$, roue dénoyée

- Démarrages à fréquence variable :

- à l'aide d'un groupe voisin (procédé “dos à dos") : groupe de Vouglans, roue noyée groupe de Sainte Croix, roue noyée groupes de Montézic (230 MW -- $428 \mathrm{tr} / \mathrm{mn}$ ) (en secours), roue dénoyée

- à l'aide d'un convertisseur statique de fréquence : groupes de Montézic, roue dénoyée.

On peut constater que tous les groupes importants sont démarrés en pompe roue dénoyée pour diminuer le couple résistant et la durée des démarrages. Ceci impose l'utilisation de matériels chers et encombrants (cuves, moto-compresseurs, tuyauteries, vannes, dispositifs de contrôle du niveau de l'eau dans les aspirateurs etc.) mais il faut remarquer que ces matériels sont nécessaires également pour le fonctionnement en compensateur synchrone.

Tous les procédés de démarrage ci-dessus donnent satisfaction, mais si les inconvénients de certains d'entre eux sont bien connus (matériel onéreux, schémas complexes, temps de démarrage assez long,etc.) il faudra attendre plusieurs années peut être pour connaître les désavantages présentés par d'autres, en particulier par le procédé asynchrone qui peut réduire sensiblement la durée de vie des alternateurs-moteurs. Rappelons à ce sujet les incidents survenus en 1975 sur plusieurs groupes bulbes de l'usine marémotrice de la Rance : après 8 ans d'exploitation il a été constaté une déformation des stators provoquée par les efforts électromagnétiques qui apparaissent au cours des régimes transitoires, particulièrement pendant les démarrages et couplages asynchrones ; cette déformation rendue possible par l'insuffisance de la fixation des circuits magnétiques sur les carcasses, a été la cause des frottements qui se sont produits entre rotors et stators.

Le procédé de démarrage le plus utilisé actuellement dans le monde est le démarrage par moteur auxiliaire asynchrone appelé couramment "moteur Poney"; le maintien du couple moteur en fonction de la vitesse se fait par variation de la résistance rotorique, soit à l'aide d'un rhéostat liquide, soit en utilisant comme à Revin un rhéostat métallique dont les éléments de résistance sont court-circuités à l'aide de contacteurs tripolaires; la variation de résistance s'opère par paliers et le réglage fin de la vitesse vers le synchronisme est obtenu par une action sur le couple résistant de la pompe-turbine (drainage de l'anneau d'eau autour de la roue).

Les démarrages asynchrones au cours desquels une énergie thermique importante s'accumule dans les pôles imposent pour les groupes de forte puissance une construction spéciale des rotors pour permettre l'évacuation des calories et de ce fait limiter l'échauffement.

Différentes techniques sont utilisées ou prévues :

Pôles feuilletés et circuits d'amortisseurs formant une cage fermée complète

- Les pôles de La Coche comportent des barres en $\mathrm{Ni}-\mathrm{Cu}$ (Monel 400) brasées à leurs extrémités sur des segments de raccordement en laiton.

- Les pôles d'Arc-Isère seront munis de barres en acier magnétique pour ne pas réduire la section de passage du flux.

\section{Pôles massifs reliés entre eux électriquement}

- Les groupes réversibles de Vouglans et Ste Croix sont munis de pôles en acier moulé.

- Le groupe III de Revin a été équipé de pôles en acier forgé en vue d'effectuer à titre expérimental des couplages en asynchrone après mise en vitesse partielle du groupe par le moteur de lancement. Les essais ont pour but de définir d'une façon précise les limites d'utilisation de ce type de pôles.

\section{Pôles feuilletés avec amortisseurs refroidis par eau}

- Le groupe IV de Revin possède des amortisseurs refroidis par de l'eau contenue dans le moyeu du rotor (rotor piscine).

\section{b) Appareillage électrique plus important et schémas d'automatisme plus complexes}

- La réversibilité des groupes conduit côté électrique à inverser deux phases sur la moyenne ou la haute 
tension, c'est-à-dire à installer un appareil électrique supplémentaire, soit à proximité de l'alternateur-moteur, soit dans le poste de raccordement au réseau H.T.

- Tous les dispositifs de démarrage, sauf s'il s'agit des alternateurs-moteurs eux-mêmes, nécessitent des appareils électriques de commande et de contrôle qui leur sont propres et, s'ils sont communs à plusieurs groupes, des câbles de raccordement aux différents alternateurs-moteurs avec des appareils de protection et de sectionnement.

- L'obligation de passer d'un mode de fonctionnement à l'autre complique les schémas d'automatisme.

A Revin on estime que par rapport à un groupe classique il y a en plus par tranche :

$25 \%$ de protections et verrouillages

$100 \%$ d'organes à commander

$500 \%$ de relais séquentiels.

Il semble qu'on soit aux limites du possible en relayage classique, et qu'il convient à l'avenir pour diminuer le taux de défaillance de l'automatisme de faire appel à des technologies nouvelles (calculateurs, microprocesseurs).

\section{c) Phénomènes transitoires - Vibrations}

Les groupes réversibles se caractérisent par des régimes transitoires au cours des périodes de démarrage, d'arrêt et de déclenchement qui donnent naissance à des écoulements très perturbés dont il faut tenir compte dans la conception et le dimensionnement de certains organes (fonds de turbines, directrices mobiles, système de commande du vannage, etc.).

Des phénomènes vibratoires dangereux peuvent parfois prendre naissance durant ces régimes transitoires en particulier au cours des emballements en turbine et des fonctionnements en pompe-frein.

Il ne semble pas actuellement que l'étude sur modèle réduit de ces régimes transitoires donne des résultats suffisants, et il en est d'ailleurs de même pour certains régimes normaux tels que les marches à faible débit et grande hauteur.

\section{d) Protections}

Les conditions d'emploi des protections électriques, mécaniques et hydrauliques des groupes binaires ne sont pas les mêmes que celles des groupes classiques : il faut en effet tenir compte de la réversibilité des groupes, du schéma électrique des installations, dispositifs de démarrage en pompe compris, et des contraintes d'ordre électrique et mécanique dues aux démarrages. Cela conduit d'une part à adapter certaines protections classiques, et d'autre part à prévoir des protections supplémentaires.

\section{Exemples d'adaptations}

- la protection différentielle longitudinale doit être verrouillée durant les périodes où des composantes apériodiques peuvent circuler au primaire des T.C. (cas des démarrages asynchrones, par moteurs auxiliaires en série avec les alternateurs et à fréquence variable);

- la protection contre les vibrations doit être également éliminée pendant les périodes de démarrage ;

-- la protection contre l'emballement doit être réglée en fonction de la plus faible des deux vitesses d'emballe. ment stabilisées et temporisées de quelques secondes.

\section{Exemples de protections spéciales}

- protections propres au dispositif de démarrage en pompe ;

- protection de décrochage en moteur : en alternateur le décrochage peut être suivi d'une resynchronisation, mais en moteur le décrochage est définitif et doit donc entraîner l'arrêt du groupe ;

- protection "temps de démarrage trop long" : les durées de démarrage en turbine et en pompe étant souvent très différentes l'une de l'autre, il est nécessaire de prévoir deux protections ou un réglage différent pour chacune des deux marches (cette protection est importante pour les démarrages qui se font roue noyée, car il y a risque d'échauffement de l'eau par barbotage) ;

- si la protection différentielle doit être neutralisée, il faut prévoir une protection spéciale de secours contre les défauts internes qui peuvent apparaître pendant le démarrage (protection à maximum de courant branchée côté réseau et suffisamment temporisée pour laisser passer les régimes transitoires).

\section{Conclusion}

Malgré les inconvénients et difficultés diverses relatives aux pompes turbines, il reste au bilan de celles-ci un avantage financier tel que leur emploi est toujours largement bénéficiaire par rapport aux groupes ternaires. Etant donné l'intérêt croissant qui va s'attacher aux stations de pompage, il convient d'attirer l'attention des constructeurs sur le fait que le maître d'œuvre est particulièrement attaché à la fiabilité des groupes, ainsi qu'à leurs possibilités d'adaptation dans les différents sites, à leur souplesse, à leur facilité d'exploitation et à leur meilleur rendement.

Parmi les problèmes qui se posent, on peut noter :

1) Est-ce qu'il existe, pour une puissance donnée, en pompage, un rapport débit turbiné/débit pompé qu'on pourrait appeler le $Q_{T} / Q_{P}$ naturel ? Et est-il possible de savoir ce que cela coûte, en argent et en points de rendement, de s'en écarter ?

2) N'y aura-t-il pas intérêt, un jour, à reconsidérer les groupes à 2 (ou pourquoi pas plusieurs) vitesses de rotation, ce qui permettrait d'adapter au mieux les puissances en pompe et en turbine? 
3) Pour les hautes chutes les maîtres d'œuvre seront toujours intéressés par les groupes à plusieurs étages réglables, pour autant bien entendu qu'ils puissent faire la preuve d'un comportement satisfaisant et d'un prix "attractif".

4) Il y a encore de nombreux points à surveiller dans la technologie des machines afin d'éviter les incidents et les défauts de jeunesse. La mise au point a quelquefois été longue, pas seulement en France d'ailleurs : il faut espérer que les expériences du passé profiteront aux projets futurs. A ce propos, on peut noter qu'il reste encore bien des options possibles, et que par exemple le mode de Aémarrage en pompe devrait être choisi non seulement en fonction de son coût propre, mais aussi en raison des répercussions sur le réseau et des fatigues imposées à la machine. On peut s'interroger aussi sur le choix du système de vannage. A l'heure actuelle les 2 solutions : cercles de vannage ou servo moteurs individuels sont utilisés concurremment dans les différentes centrales, sans parler de Revin qui est un système intermédiaire. Il serait bon un jour de savoir quelle est la solution préférable.

5) Il faut insister sur le fait que les pompes-turbines et leurs alternateurs-moteurs, sont des machines très sollicitées. Le nombre de mises en route, arrêts, changements de marche, est très élevé ; de plus des régimes instables soumettent le matériel à des contraintes vibratoires sévères.

Pour avoir une bonne fiabilité, il ne faut pas hésiter à prendre des précautions pouvant paraître surabondantes et par exemple consentir une perte de rendement de 1 ou 2 points, pour adopter une machine plus sûre.

Les 2 tableaux ci-après indiquent la puissance et la vitesse spécifique en fonction de la hauteur de chute des principales pompes-turbines mondiales :

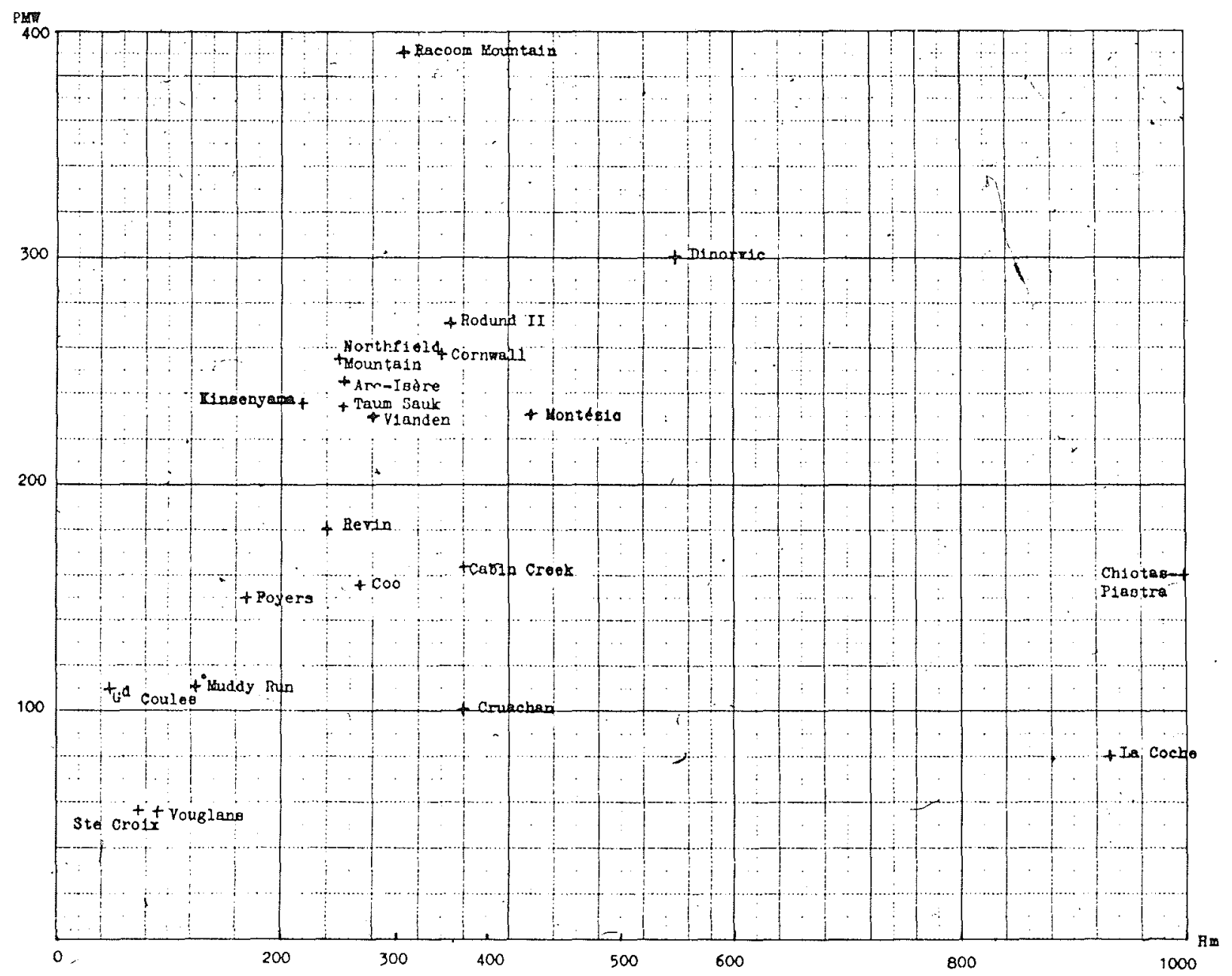

Caractéristiques des principales pompes-turbines (Pmax - H) 

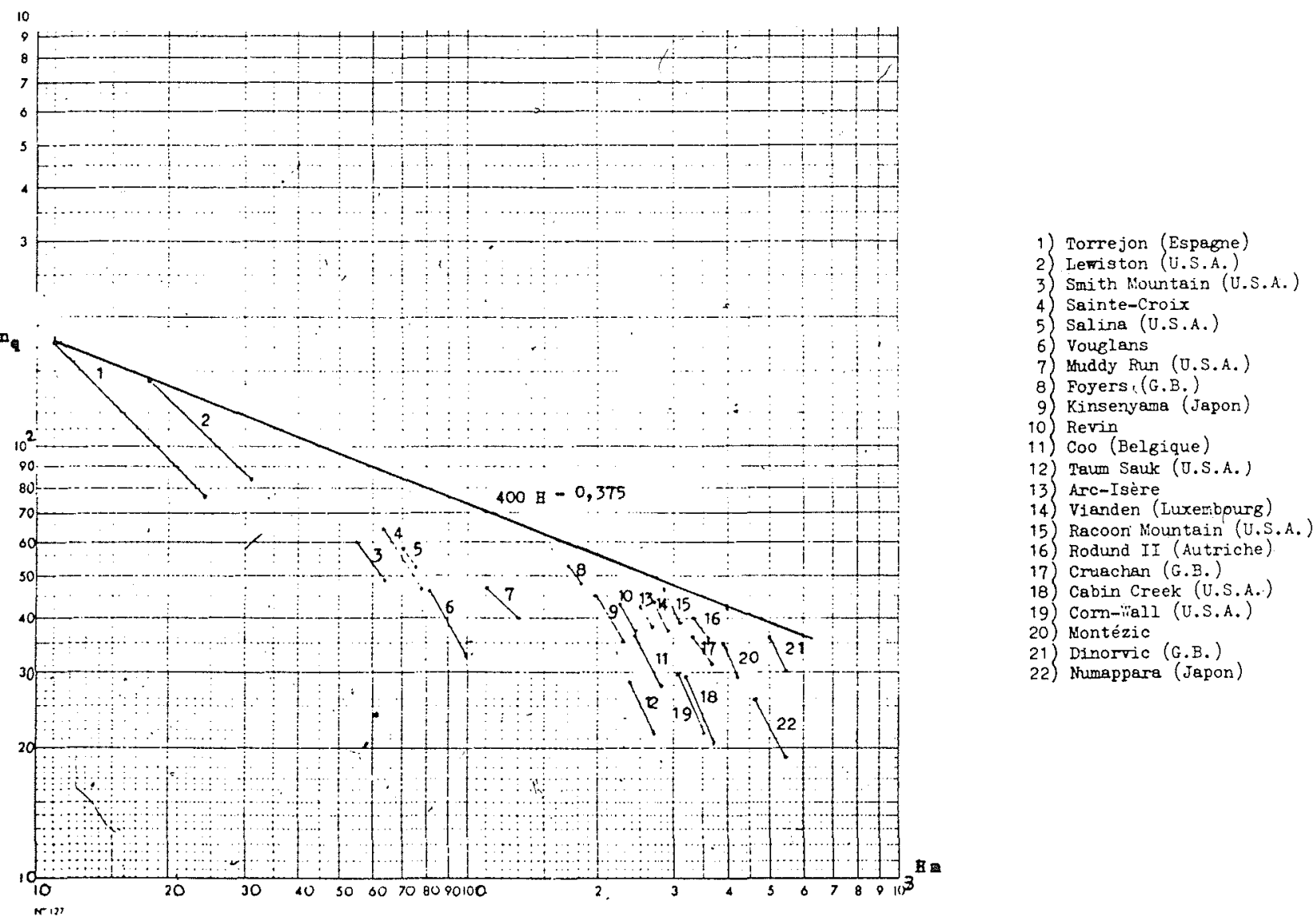

Caractéristiques des principales pompes-turbines (nq-H)

\section{Discussion}

Président : M.C. GEMAEHLING

M. le Président remercie M. PLICHON de son très in téressant exposé sur le problème des turbines-pompes qui est tout à fait d'actualité et d'un intérêt économique considérable. Il ouvre sans plus tarder la discussion:

Un échange de vue s'instaure entre M. BOUSSUGES (Neyrpic, Grenoble) et M. PLICHON sur la valeur du rapport :

$$
\frac{\mathrm{Qt}}{\mathrm{Qp}}=\frac{\text { Débit maximum turbiné }}{\text { Débit maximum pompé }}
$$

dans diverses installations déjà réalisées.

II en ressort que ce rapport, voisin de 1,30 dans les exemples donnés, dépend du tracé de la roue, de son nombre de tours spécifique et de divers autres paramètres.

L'analyse de cette question trouvera sa place au cours des discussions des mémoires présentés dans la séance de l'aprèsmidi, conclut $M$. le Président.

M. LACOSTE s'interroge sur le rôle des stations de pompage en liaison avec certaines servitudes d'exploitation des centrales nucléaires, au cours d'une intervention qu'il a résumée comme suit :

Les besoins en "puissance réglante" augmenteront, à moyen terme, avec la croissance de la part de puissance d'origine nucléaire sur le réseau. Or, les centrales nucléaires fonctionnent actuellement et fonctionneront pour les centrales en construction, à puissance constante sans aucune possibilité de modulation rapide permettant le réglage primaire ou secondaire. Peut-on espérer que les centrales de pompage aideront à résoudre ce problème et comment? Les groupes sans réglage ne présentent-ils pas de ce fait, un grave handicap?

Ces groupes, répond M. PLICHON, gardent toujours l'intérêt de concourir au passage des périodes de pointe d'hiver et de diminuer, de ce fait, la puissance qu'on aurait dû installer dans des usines génératrices classiques à cet effet. Dans l'avenir, il pourrait peut-être être complétés par des dispositifs susceptibles de les faire participer au réglage.

See English abstract on next page 


\section{Abstract \\ A Project Owner's view of the pump-turbine problem. Technical and economic aspects}

With the present considerable expansion of energy transfer by pumping, Electricite de France frequently has to compare several alternatives, among which especially ternary and reversible power units. Technical and cost comparisons for these two alternatives are the subject of the first part of this report.

In the second part of the report, problems peculiar to reversible pumps are discussed, which are mainly connected with pump start-up methods, electric circuitry and equipment, transient effects, and protective devices or systems.

Unit output and specific speed data for the most important pump-turbines in the world are shown separately in terms of head on two graphs at the end of the report. The present specific speed limit appears to be denoted by a curve of the form $400 \mathrm{H}-0.375$. 